\title{
A Fast-Sampling, Fixed Coil Array for Measuring the AC Field of Fermilab Booster Corrector Magnets
}

\author{
J. DiMarco, D. J. Harding, V. Kashikhin, S. Kotelnikov, M. Lamm, A. Makulski, R. Nehring, D. \\ Orris, P. Schlabach, W. Schappert, C. Sylvester, M. Tartaglia, J. Tompkins, and G.V. Velev \\ Fermi National Accelerator Laboratory, Batavia, IL, 60510, USA
}

\begin{abstract}
A system employing an array of inductive pick-up coils around the perimeter of a cylinder has been developed for measurements of the rapidly changing field in the new corrector magnets for the Fermilab Booster. The coils are fabricated on printed circuit boards and feature windings which buck dipole, quadrupole, and sextupole fields, allowing sensitive measurements of both strength and higher-order harmonics. The array of coils is simultaneously sampled at data rates of up to $100 \mathrm{kHz}$ with $10 \mathrm{kHz}$ bandwidth using 24-bit ADC's.
\end{abstract}

Index Terms - Fast magnetic field measurements, circuit board magnet measurement probes, AC field measurements.

\section{INTRODUCTION}

$\mathrm{N}$ EW corrector magnets are being built at Fermilab for the Booster ring. These will for allow correction of orbits and tunes through the entire Booster cycle to maximize proton delivery for neutrino experiments. The corrector package includes six different corrector elements, providing normal and skew orientations of dipole, quadrupole, and sextupole. Each of these elements can be independently powered. A more detailed description of the innovative magnet design is given elsewhere [1]. The magnets will operate in the Tevatron Booster ring, cycling at a rate of $15 \mathrm{~Hz}$. Furthermore there is a beam transition region where some elements change from full positive to full negative field in 1 millisecond. Table 1 shows the maximum field and field slew rates for each corrector. A total of 60 magnets will be tested and characterized to obtain strength and harmonics data for beam simulations. The ability of the field to follow the current and the presence of eddy current and coupling effects in the elements will also be tested. The magnets require measurements performed at sampling rates of at least $10 \mathrm{kHz}$ through at least the first allowed harmonic of each element. With the sextupole elements, this implies harmonics through 18-pole. The mechanical length of the magnet is $425 \mathrm{~mm}$. However, the large, $138 \mathrm{~mm}$, aperture causes the end field to extend considerably beyond the physical magnet assembly.

Manuscript received August 27, 2007. This work was supported in part by the U.S. Department of Energy .

All authors are with Fermi National Accelerator Laboratory, Batavia, IL, 60510, USA (corresponding author is Joseph DiMarco phone: 630-840-2672; fax:630-840-8079; e-mail: dimarco@fnal.gov).
TABLE 1 Field Parameters for the Booster Corrector Magnets

\begin{tabular}{ccc}
\hline \hline $\begin{array}{c}\text { Corrector } \\
\text { Type }\end{array}$ & $\begin{array}{c}\text { Maximum Integral } \\
\text { Field } \\
\text { at Full Current }\end{array}$ & $\begin{array}{c}\text { Maximum } \\
\text { Integral Field } \\
\text { Slew Rate }\end{array}$ \\
\hline Horizontal Dipole & $0.015 \mathrm{~T}-\mathrm{m}$ & $3.5 \mathrm{~T}-\mathrm{m} / \mathrm{s}$ \\
Vertical Dipole & $0.015 \mathrm{~T}-\mathrm{m}$ & $3.5 \mathrm{~T}-\mathrm{m} / \mathrm{s}$ \\
Normal Quad & $0.16 \mathrm{~T}$ & $160 \mathrm{~T} / \mathrm{s}$ \\
Skew Quad & $0.008 \mathrm{~T}$ & $0.8 \mathrm{~T} / \mathrm{s}$ \\
Normal Sextupole & $1.41 \mathrm{~T} / \mathrm{m}$ & $2279 \mathrm{~T} / \mathrm{m} / \mathrm{s}$ \\
Skew Sextupole & $1.41 \mathrm{~T} / \mathrm{m}$ & $2279 \mathrm{~T} / \mathrm{m} / \mathrm{s}$ \\
\hline \hline
\end{tabular}

In order to measure the multipole fields of these relatively weak, fast-ramping corrector magnets, two parallel measurement methods were pursued. The first featured a standard harmonics coil rotating slowly with its period synchronized to the AC excitation cycle of the power supplies [2]. The second system developed was a fixed (i.e. nonrotating) coil system, with multiple identical probes distributed at various angles being sampled simultaneously by high-speed electronics. This fixed-coil system and a first look at its performance are described in this paper.

\section{MeAsurement Technique}

The Fixed-Coil Probe Array (FCPA) consists of 32 inductive pick-up coils fabricated on printed circuit boards and positioned on a cylindrical form. Simultaneous sampling of the field using printed circuit pick-up coils has been employed with success by BNL for measurements of the GSI magnets [3]. A fundamental challenge in this technique is the dynamic range required by electronics to satisfactorily resolve small harmonic signals measured together with the fundamental field. This resolution is typically required to be better than 10$100 \mathrm{ppm}$. Mechanically, the errors in placement of the coils, coupled with the large fundamental field, can also give rise to spurious harmonics because these errors act effectively as torsional and transverse vibrations [4]. Though in general, it is possible to remove the false-harmonic effects with calibration.

For our FCPA, we developed pick-up coils which suppressed the fundamental field. This was intended to address both of the above issues associated with the 
simultaneously sampled pick-up coil technique. With the main field largely removed, the dynamic range required drops accordingly with the ratio of suppression (i.e. with bucking ratio). Likewise, the effects of mechanical errors are also diminished by the bucking ratio, so that, e.g., coil misplacements of 100 microns become effectively 1 micron for bucking ratio of 100 . The pick-up coils are fabricated from circuit boards in order to achieve a high uniformity among the vertices along with robustness and low cost. The suppression of the main field(s) is achieved by employing radial bucking, and consequently, the circuit board plane containing the coil windings is attached radially to the cylindrical form (see Figure 1). The bucking is achieved locally - each vertex independently bucks the main field - the uniformity of the bucking among the probes therefore also is relevant.

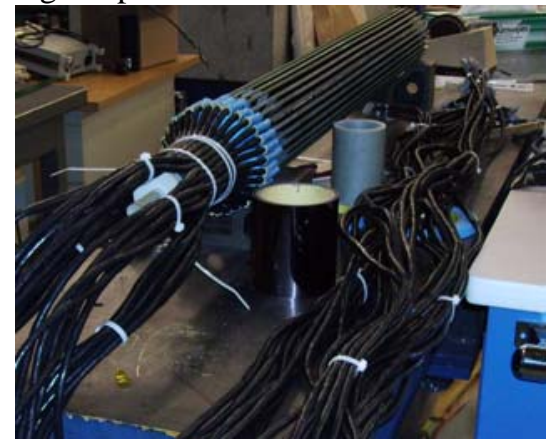

Figure 1 . The fixed coil probe with 32 radially mounted circuit board probes.

\section{A. Circuit Board Probes}

The circuit boards fabricated for the fixed-coil probe are $1.016 \mathrm{~m}$ (40 in.) in length, long enough to capture the full integral field to better than a couple of percent (estimated from calculation). The chosen length was a compromise in achieving as long a board as possible within reasonable cost. The boards have 22 layers; 20 of which are active, with the outside 2 layers used only for interconnections. The 22 layers comprise about $3 \mathrm{~mm}$ of the $5 \mathrm{~mm}$ total board thickness, since there is a $1 \mathrm{~mm}$ cladding on each side of core. The boards are about $26.5 \mathrm{~mm}$ wide with additional $\sim 6 \mathrm{~mm}$ extension tabs located at several locations which are used to register the boards in the cylindrical form. The axial locations of the tabs are the only difference between two alternating types of boards which populate the form. The tabs are shifted between the two types in order to achieve nearly contiguous placement of the boards on the form without compromising the form integrity. The form diameter is about $58 \mathrm{~mm}$, and total FCPA diameter is $111 \mathrm{~mm}$. This is small enough to measure the magnet with its $115 \mathrm{~mm}$ ID beam position monitor inserted.

The trace pattern for the first two active layers is shown in Figure 2. The trace width is $0.1 \mathrm{~mm}$ and traces are separated by $0.15 \mathrm{~mm}$ gaps. The four identical 'loops' of a layer have 9 turns each. Combining loops 1 and 3 (or loops 2 and 4), which have opposite chirality, creates a signal in which the dipole is bucked (DB signal). To create a signal in which both dipole and quadrupole are bucked, the loop 1/3 and loop 2/4 dipole buck combinations are themselves combined (DQB signal). Furthermore, since the four identical loops of the odd layers also appear on the even layers but at a different radial position, the odd and even layer dipole-quadrupole bucked signals can in turn be combined to form a signal which has dipole, quadrupole, and sextupole bucking (DQSB signal).

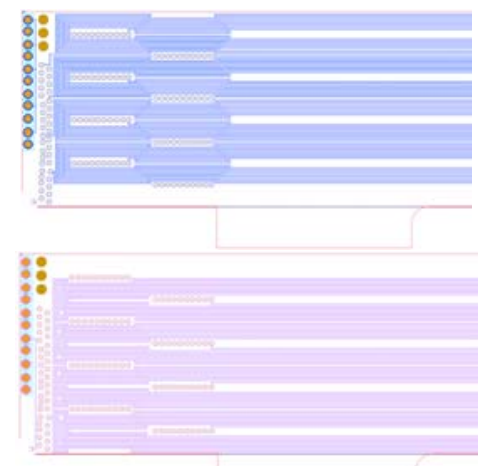

Figure 2. The connection end regions of the circuit board layer 1 (top) and layer 2 (bottom) showing the 8 blocks of conductor 'loops' of the odd and even probe layers. Loops 1-8 refer to loops shown from top to bottom.

In addition to the bucked signals, two unbucked signals are also available on the probe. The first layer of loop 1 by itself forms the 'unbucked low-gain' (UBL) signal, insuring that there are signals which do not saturate the ADC's. The sum of loop 1 through the 10 even layers forms the 'unbucked highgain' (UBH) signal. Layers are connected by 'thru' vias in order to reduce cost. All traces on the board are connected serially, so that there is only one electrical net. The total length of the trace is about $1.44 \mathrm{~km}$. ( 0.9 miles). The boards were fabricated by Sanmina Corp. in California at unit cost of roughly $\$ 400$ for 44 boards. Sensitive resistance tests done during production help insure absence of shorting within loops. With 9 turns per loop, 1 shorting turn results in about $10 \%$ resistance change for that layer. Resolution was at the few percent level, including natural resistance variation in the material/etching. Nominal resistance was $180 \mathrm{Ohms}$ for a 9turn, 1-layer loop, and therefore about $14.4 \mathrm{kOhm}$ for the entire circuit. The manufacturer worked closely with us in establishing and maintaining good quality testing metrics.

The probes were mounted by inserting the tabs with thin Kapton shims into the machined grooves on the cylindrical form and using a quick-cure epoxy at non-groove locations to anchor the probes to the form. This allows removal and replacement of probes if necessary. Slot size tolerance of the form grooves is about $0.25 \mathrm{~mm}$. With radial mounting of the boards, however, the position error including angular play within the slot was $1 \mathrm{~mm}$ at the far edge. Based on studies with prototype boards having $0.6 \mathrm{~m}$ length, there was no real concern that this would affect the results of fundamental or bucked harmonic field measurements. Simulations with random board placement errors of this magnitude were also consistent with this.

\section{B. Data Analysis}

Since the output of the data acquisition system yields flux as a function of angle, and the probes are quasi-identical (i.e. effectively the same probe is at the various angular positions), data can be treated as simply coming from a single rotating 
circuit board probe. Though the conductor geometry on the radially-bucked circuit board probes is relatively complex, from the point of view of a generalized approach to rotating probe harmonics analysis, it is not exceptional. The angular flux can be expressed as

$$
\phi(\theta)=\mathfrak{R} \sum_{n=1} C_{n} K_{n} e^{(i n \theta)}
$$

where the field is defined as

$$
C_{n}=B_{n}+i A_{n}
$$

and the winding sensitivity is given by the sum over all wires on the probe

$$
K_{n}=\sum_{j=1}^{N_{\text {wires }}} \frac{L_{j} R}{n}\left(\frac{\left(x_{j}+i y_{j}\right)}{R}\right)^{n}(-1)^{j}
$$

Here $L$ is the length of a given wire and $R$ is the reference radius. The $(-1)^{j}$ gives the sign of the current flow of each wire and the $\left(x_{j}, y_{j}\right)$ are the locations of the wires with respect to the rotation axis. The above parameters for each wire on the probe are listed in a standard coil parameter file. The large number of wires (e.g. 1440 for the DQS bucked signal) is transparent to the sensitivity calculation performed on the file. Wire position parameters include the small offsets caused by the lateral positioning of the various layers within the probe thickness. The harmonic fields can be determined by obtaining the complex Fourier coefficients $\boldsymbol{F}_{\boldsymbol{n}}$ from an FFT of the measured data, and then dividing by the complex sensitivities. That is,

$$
C_{n}=\frac{F_{n}^{*}}{K_{n}}
$$

where $\boldsymbol{F}_{\boldsymbol{n}}{ }^{*}$ is the complex conjugate of $\boldsymbol{F}_{\boldsymbol{n}}$. This is the same analysis which can be applied to all rotating coil data [5].

\section{DATA ACQUISITION SYSTEM}

A schematic of the data acquisition electronics is shown in Figure 3. The FCPA signals travel through three stages. The first is the Fixed Coil Interface Box which routes the 5 coils of each of the 32 probes (160 signals) to create 5 mass termination connectors for each of the UBL, UBH, DB, DQB, DQSB array signals. Additionally, it outputs a cable ID number to the DAQ system so that the configuration can be automatically recorded.

The second stage is the Signal Conditioner (SC) which acts as both pre-amplifier with programmable gains as well as converting the signals from true differential inputs to singleended outputs. To lower the noise and select the appropriate bandwidth, an additional RF filter and LP filter are also part of the SC modules. An input buffer amplifier prior to RF filter is used to deal with the various and large (as much as $14 \mathrm{kOhm}$ ) resistances of the probe signals. The SC has a floating analog ground with only one connection (which is at the ADC). The modules also have low cross-talk between channels. The available gains with at least $10 \mathrm{kHz}$ bandwidth are 1, 10, 100 .

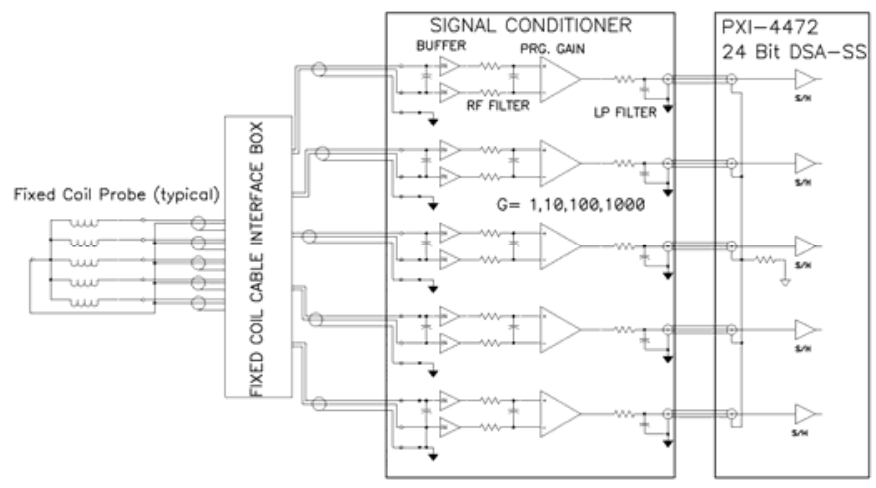

Figure 3. Fixed Coil Signal Layout: illustrating the signal paths for a typical probe on the array.

The last stage in the acquisition path are the National Instruments NI PXI-4472B ADC modules (Dynamic Signal Analyzer (DSA) modules), which simultaneously sample 8 channels of input and have 24 bit Delta Sigma ADC resolution with dynamic range of $110 \mathrm{~dB}$. The sampling can be synchronized by an external trigger or software internal (bus) trigger.

Data acquisition and control is run from a LabView based GUI. A user definable set of current profiles played through an NI PXI-6673 8-channel, 16-bit DAC generates the waveforms used to excite the magnet power supplies. Any combination of arbitrary current ramps on any arbitrary combination of power supplies can be excited. Each DSA is triggered synchronously with the current profiles applied to the magnet coils, and after triggers are collected, the data is averaged and integrated in time to recover flux as a function of time. The offset of each ADC channel is corrected for drift and the averaged, corrected, data are passed to the Extensible Measurement System (EMS) [6] package, for processing of magnetic field results. These are then returned to the LabView control system for display to the operator, and sent to an archiving system for storage via a web service interface.

\section{Results}

The fixed coil system began taking data in middle July 2007, so data presented here reflect first results. Since the power cycling is occurring at the rate of $15 \mathrm{~Hz}$, measurements of strength and harmonics for the 6 correctors are acquired very rapidly with tests being completed in less than 30 minutes including switching between various correctors. Typically 100 cycles are sampled and averaged (about 6 seconds of data). A sample measurement of field is shown in Figure 5. Ability of field to track current is shown in Figure 6; the field difference seen during the fast-ramping portions corresponds to about $20 \mu \mathrm{s}$ lag. Note that time resolution is 10 $\mu$ s per sample and that data have very little noise even at the $0.1 \mu \mathrm{T}$-m level 

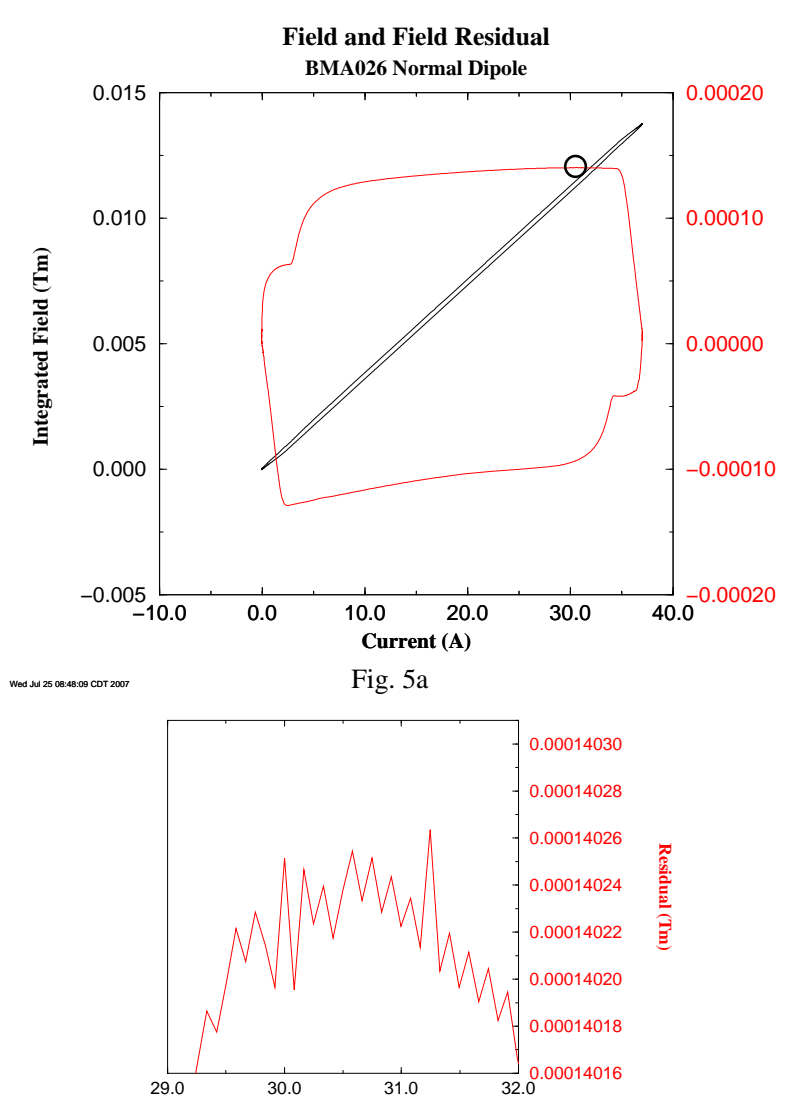

Fig. $5 b$

Figure 5. Field Strength vs. Current is shown in 5 a along with the non-linear field residual. The circled area in $5 a$ is expanded in $5 b$ to show the level of field resolution in the fixed coil measurements.

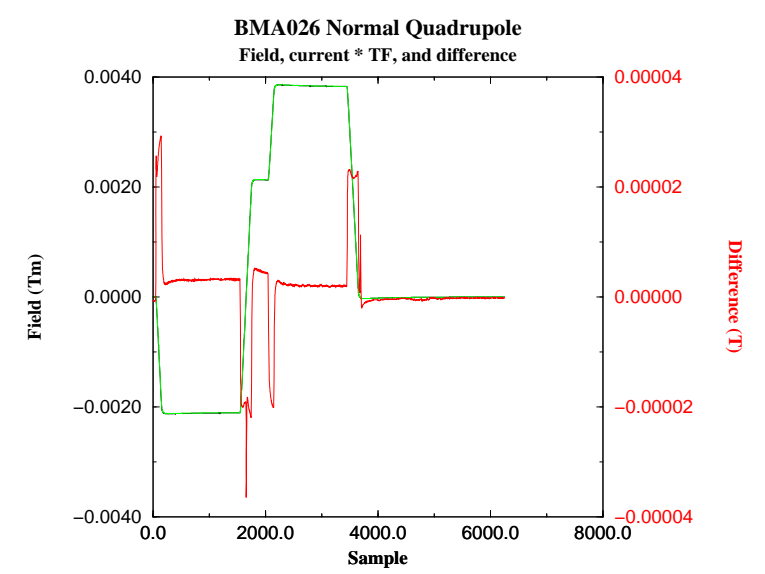

Figuresesaranthe meas. field and calculated field (from current times transfer function) are over plotted. Their difference is also shown (right-hand axis).

Bucked signals from the flattop portion of the ramp are shown in Figure 7. The signals show very little fundamental fields and are dominated by the high order allowed harmonics (22 pole, 20 pole and 18 pole). Bucking ratios are about 1500 for the array as a whole. A sample of the field shape reconstruction is shown in Figure 8. One can see that the error fields are small (less than $0.0001 \mathrm{~T}-\mathrm{m}$ ) out to at least $+/-37.5$ $\mathrm{mm}$.

\section{CONCLUSION}

A fixed coil probe has been developed for measuring the AC fields of the new Fermilab Booster corrector magnets. The

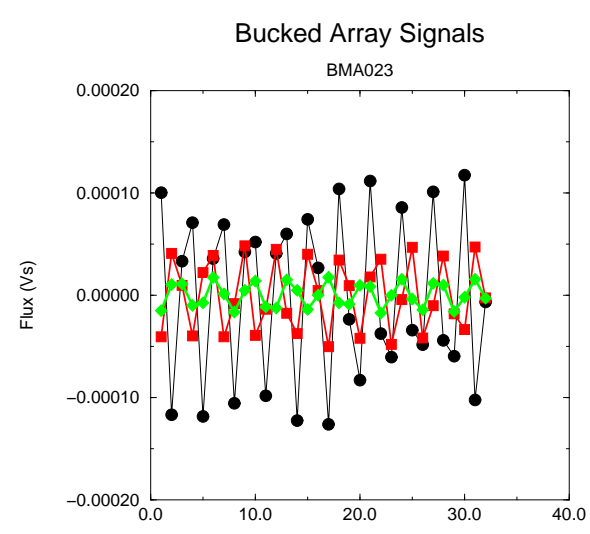

Figure 7. Bucked signals from dipole, quad., and sextupole measurements.

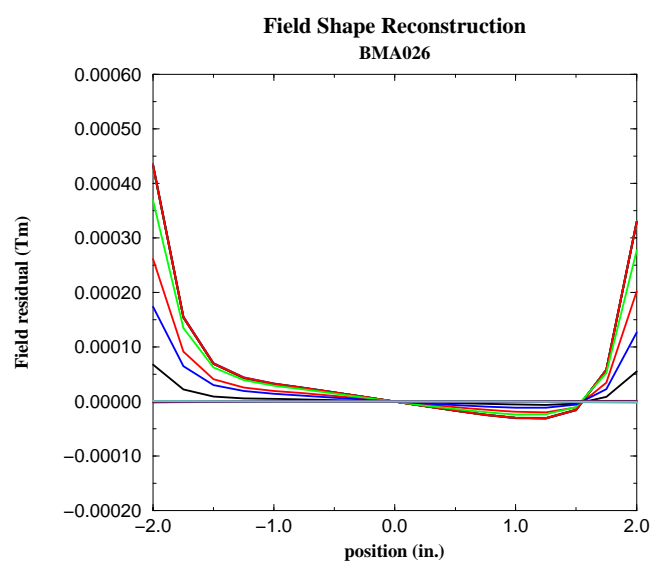

Figure 8. Field shape reconstructed from measured multipoles shown every 2 milliseconds (the available data has been decimated for plotting clarity).

probe data have excellent time and field resolution , and show close tracking of current and field within 20-30 $\mu$ s. Magnet total harmonics shape deviation at $\pm 37.5 \mathrm{~mm}$ is also less than $0.0001 \mathrm{~T}-\mathrm{m}$, indicating low eddy-current effects.

\section{ACKNOWLEDGMENT}

The contributions of MTF technicians and staff are gratefully acknowledged, as are those of Clark Reid for mechanical design and Tom Wesson and Johnny Green for circuit design and procurement. Also, our thanks to Sanmina Corporation for their cooperation and quality work.

\section{REFERENCES}

[1] V.S. Kashikhin, et al., "A New Correction Magnet Package for the Fermilab Booster Synchrotron,” PAC’05, May 2005

[2] G.V. Velev, et al., "A slowly rotating coil system for AC field measurements of Fermilab Booster correctors”, PAC’07, June 2007

[3] P. Wanderer, et al., "Development of a Precise Magnetic Field Measurement System for Fast-Changing Magnetic Fields", IEEE Trans. Appl. Superconductivity, Vol. 14, No. 2, June, 2004.

[4] J. DiMarco et al., "Influence of Mechanical Vibrations on the Field Quality Measurements of LHC Interaction Region Quadrupole Magnets”, IEEE Trans. on Appl. Supercond., Vol. 10, No. 1, Mar. 2000.

[5] J.DiMarco, T. Ogitsu, "JAVA APIs for Field Meas. Analysis”, unpub.

[6] J. Nogiec, et al., "A Configurable Component-Based Software System for Magnetic Field Measurements”, IEEE Trans. Appl. Superconductivity, Vol. 16, Issue 2, June 2006, Page(s):1382 - 1385. 